\title{
The decapentaplegic gene is required for dorsal-ventral patterning of the Drosophila embryo
}

\author{
Vivian F. Irish ${ }^{\mathbf{1}}$ and William M. Gelbart \\ Department of Cellular and Developmental Biology, Harvard University, \\ Cambridge, Massachusetts 02138-2097 USA
}

\begin{abstract}
The decapentaplegic gene (dpp), which encodes a growth factor-like protein (Padgett et al. 1987), is implicated in several morphogenetic events in Drosophila melanogaster. We define here a novel embryonic function encoded by $d p p^{\text {Hin }}$ alleles of the $d p p$ gene. $d p p^{\text {Hin }}$ null homozygotes die as ventralized embryos. $d p p^{\text {Hin }}$ activity is not required in the maternal germ line since lack of $d p p^{H i n}$ function during oogenesis has no effect on the zygotic phenotype. Since $d p p^{H i n}$ null embryos are already abnormal early in gastrulation, the $d p p^{H i n}$ product is an early-acting, strictly zygotic function involved in establishing the embryonic dorsal-ventral pattern. Several maternally acting dorsalizing genes are thought to be required for the establishment of a dorsal-ventral morphogenetic gradient (Anderson et al. 1985b). We have examined the interactions of $d p p^{\text {Hin }}$ mutations with three of these genes. Embryos null for $\boldsymbol{d p} p^{\mathrm{Hin}}$ and derived from a mother homozygous for a dorsalizing mutation exhibit a lateralized phenotype, indicating that the dorsal-ventral identity of the epidermis in part derives from the direct or indirect regulation of $\boldsymbol{d p p} p^{H i n}$ activity by these genes.
\end{abstract}

[Key Words: Decapentaplegic; dorsal-ventral patterning; Drosophila; embryogenesis]

Received April 22, 1987; revised version accepted August 5, 1987.

The establishment of the embryonic body pattern in Drosophila melanogaster depends on the complex interplay between maternal products and zygotic genes. A number of maternally acting genes required for the formation of the embryonic dorsal-ventral pattern have been identified and characterized (Anderson and Nüsslein-Volhard 1984a,b). A loss-of-function mutation in any of these loci results in a dorsalized embryonic phenotype in which the embryo consists of an elongate tube covered with dorsal cuticle. This alteration is due, at least in the case of dorsal, to a shift in the dorsal-ventral pattern such that all cells acquire a more dorsal identity (Nüsslein-Volhard et al. 1980). Several biochemical and genetic studies have begun to define the maternal gene products required to establish the embryonic dorsalventral pattern (Anderson and Nüsslein-Volhard 1984a; Anderson et al. 1985a). However, only a few zygotic genes involved in interpreting this information have been identified. These include zerknüllt, tolloid, and shrew, which are required for the formation of dorsally derived tissues (Jurgens et al. 1984; Wakimoto et al. 1984), and twist and snail, which are necessary for the formation of the ventrally derived mesoderm (Simpson 1983; Nüsslein-Volhard et al. 1984). Here we define a novel zygotic function encoded within the $d p p^{H i n}$ region

'Present address: Department of Genetics, University of Cambridge, Cambridge CB2 3EH, UK. of the decapentaplegic gene $(d p p)$ and show that this region is required for dorsal epidermal tissue formation in the embryo.

Our earlier studies on dpp have defined three functionally distinct regions: $d p p^{d i s k}, d p p^{s h v}$, and $d p p^{H i n}$ (Fig. 1) (Spencer et al. 1982; Segal and Gelbart 1985; note our revised nomenclature described in Materials and methods). Functions encoded in the imaginal disk specific $\left(d p p^{d i s k}\right)$ portion are required for normal adult pattern formation (Spencer et al. 1982). Mutations in the $d p p^{\text {disk }}$ region principally result in deletion of pattern elements of the adult epidermal tissues derived from the imaginal disks. In general, increasingly severe $d p p^{d i s k}$ mutations result in the loss of correspondingly more imaginal disk-derived tissue. The relationship of the deleted pattern elements to their imaginal disk fate map positions has led to the suggestion that the $d p p^{d i s k}$ region may be involved in positional specification along the proximo-distal axis of the developing imaginal disks (Spencer et al. 1982). Mutations in the shortvein $\left(d p p^{s h v}\right)$ region elicit head and wing venation defects, and most $d p p^{s h v}$ alleles result in larval lethality (Segal and Gelbart 1985). Lying between the $d p p^{s h v}$ and $d p p^{d i s k}$ regions is a haplo-lethal region termed $d p p^{\text {Hin }}$ (Haplo-insufficiency near decapentaplegic; Spencer et al. 1982; Segal and Gelbart 1985). Animals carrying only one wild-type copy of this region die during embryogenesis. Two recessive embryonic lethal $d p p$ alleles have been identified on the 

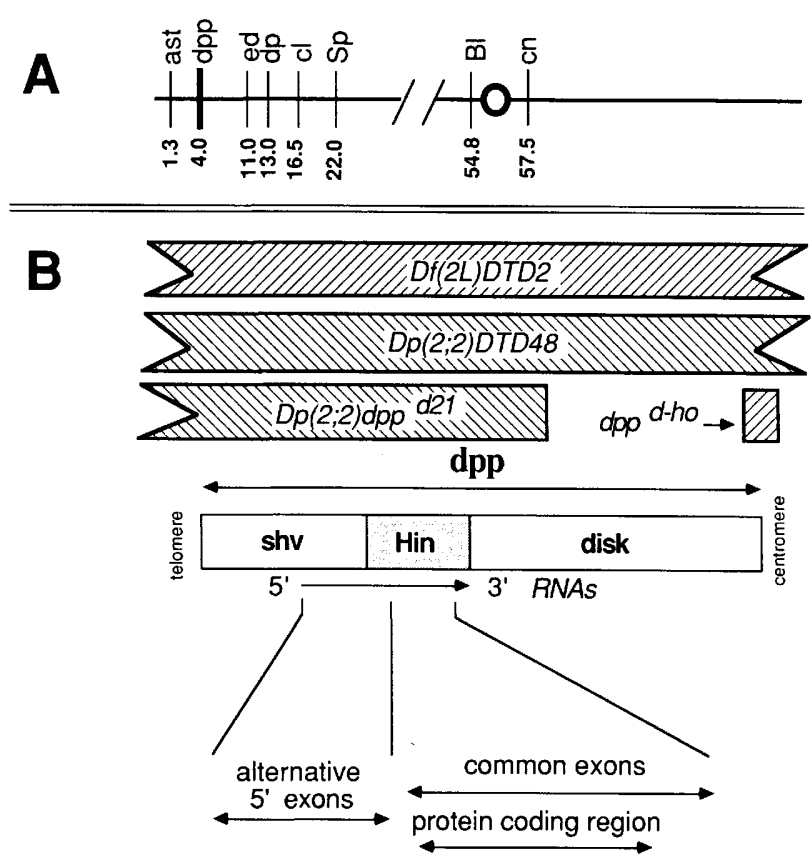

Figure 1. Chromosomal maps of the decapentaplegic $(d p p)$ region. (A) A linkage map of the second chromosome, indicating the recombinational positions of several flanking markers employed in these experiments (see Materials and methods) (Lindsley and Grell 1968). (B) A genetic and molecular map of the $d p p$ gene. $d p p$, located distally on the left arm of chromosome 2 in polytene region $22 \mathrm{~F} 1-2$, has been subdivided into three regions based on genetic and molecular criteria (Spencer et al. 1982; Segal and Gelbart 1985; Gelbart et al. 1985; see Materials and methods for revisions to $d p p$ nomenclature). Above the map are noted the locations and extents of four rearrangements used in the present studies. $d p p^{d-h o}$ is a $2.7-\mathrm{kb}$ deletion (Blackman et al. 1987) that confers a recessive heldout wing phenotype. $D p(2 ; 2) d p p^{d 21}$ and $D p(2 ; 2) D T D 48$ are two $d p p^{\text {Hin }}+$ duplications that lack $d p p^{h o+}$ function, whereas $D f(2 L) D T D 2$ removes the entire $d p p$ gene and surrounding sequences. Below the map, the orientation and extent of several species of polyadenylated $d p p$ transcripts are noted. These transcripts share exons derived from the $d p p^{H i n}$ region, but possess different 5'-untranslated exons (St. Johnston and Gelbart 1987; Padgett et al. 1987; R.D. St. Johnston, pers. comm.).

basis of allelism to $d p p^{d i s k}$ lesions; by complementation mapping, they are inseparable from the $d p p^{\text {Hin }}$ region (Spencer et al. 1982). These two recessive lethal mutations (which we will refer to as $d p p^{\text {hin-r }}$ mutations) are allelic to most $d p p^{d i s k}$ and $d p p^{\text {shv }}$ lesions; occasional cases of partial complementation have been shown to be synapsis-dependent (Gelbart 1982; Wu 1984).

We describe the isolation of dominant haplo-lethal $d p p^{\text {Hin }}$ mutations and the embryonic phenotypes produced by these mutations as well as by recessive embryonic lethal $d p p$ lesions. Complete loss of $d p p^{H i n}$ activity results in a striking "ventralized" embryonic phenotype, in which the normally ventral epidermal pattern elements are present both ventrally and dorsally. We present experiments indicating that this phenotype is due to strictly zygotic expression. The $d p p^{\text {Hin - null phe- }}$ notype is opposite to that produced by loss-of-function lesions in maternally expressed "dorsalizing" genes, which result in the replacement of ventral with dorsal pattern elements (Anderson and Nüsslein-Volhard 1984b). We have examined the phenotypes of animals lacking maternal activity for any of three dorsalizing genes as well as for zygotic $d p p^{\text {Hin }}$ expression. The lateralized phenotypes of embryos of these double-mutant combinations demonstrate that embryonic lateral epidermal tissue can be formed, even in the absence of the maternal dorsalizing gene products. These results, together with observations on in situ patterns of embryonic expression of $d p p$ transcripts (St. Johnston and Gelbart 1987), suggest that $d p p^{\text {Hin }}$ activity is required early in development for formation of dorsal epidermal tissue.

\section{Results}

Generation of $\mathrm{dpp}^{\mathrm{Hin}}$ mutations

$d p p^{\text {Hin }}$ mutations were generated utilizing three different protocols (see Materials and methods). Because deficiencies that remove the $d p p^{H i n}$ region display dominant embryonic lethality, all three schemes incorporated an extra copy of $d p p^{H i n+}$ so that potentially haplolethal mutations would be recoverable among some progeny classes. One protocol was in essence an $F_{2}$ lethal screen focusing on mutations allelic to a tester recessive $d p p^{\text {Hin }}$ mutation.

The other two protocols were $F_{1}$ screens for morphological mutations (heldout wings), which took advantage of the following observations. Given the similarity in lethal phenotype between homozygotes for the recessive embryonic lethal $d p p$ alleles and monosomics for $d p p^{\mathrm{Hin+}}$, Spencer et al. (1982) suggested that these recessive alleles are leaky $d p p^{\text {Hin }}$ mutations. The recessive embryonic lethal alleles partially inactivate functions of the $d p p^{d i s k}$ region. If these recessive embryonic lethal $d p p$ alleles do indeed represent hypomorphic mutations of the haplo-lethal function, we inferred that, in the presence of $d p p^{\text {Hin }+} d p p^{\text {disk- }}$ duplications, dominant haplo-lethal mutations could be recovered on the basis of $F_{1}$ adult $d p p^{\text {disk }}$ phenotypes.

Using both the $F_{1}$ and $F_{2}$ protocols, $20 \mathrm{dpp}$ lesions were identified (Table 1). Three are standard mutations in the $d p p^{\text {disk }}$ region, whereas another eight are gross deletions of $d p p$. Nine behave as if they are lesions within the $d p p^{H i n}$ region. One of these nine alleles, $d p p^{e 87}$, completely complements mutations of the $d p p^{\text {shv }}$ and $d p p^{d i s k}$ regions and therefore is given a special designation $\left(d p p^{e}\right.$ for embryonic). The other eight behave as if they partially or fully inactivate the functions of these other regions of $d p p$.

\section{Embryonic phenotypes elicited by dpp ${ }^{\mathrm{Hin}}$ mutations}

The cuticular pattern generated during embryonic development contains a number of recognizable structures that define both the position and the polarity of the underlying epidermis (Fig. 2A,B). Although the cuticle is not deposited until late in embryogenesis, its morphology can reflect a variety of pattern alterations caused by many maternal-effect and early-acting zygotic mutations. Below, we describe the different mutant cu- 
Table 1. dpp lesions generated in this study

\begin{tabular}{|c|c|c|}
\hline Mutant lesion & Protocola $^{a}$ & Cytology \\
\hline$D f(2 L) d p p^{\text {Hin } 32}$ & 1 & $\mathrm{Df}(2 \mathrm{~L})$ 22E3-F1; 23Al-2 \\
\hline$D f(2 L) d p p^{\text {Hin } 34}$ & 1 & $\mathrm{Df}(2 \mathrm{~L}) 22 \mathrm{E} 2-3 ; 23 \mathrm{~A} 2-4$ \\
\hline $\operatorname{In}(2 L R) d p p^{d 35}$ & 1 & $\operatorname{In}(2 \mathrm{LR}) 22 \mathrm{~F} 1-2 ; 42 \mathrm{~A} 2-8$ \\
\hline $\operatorname{In}(2 L) d p p^{d 36}$ & 1 & $\operatorname{In}(2 \mathrm{~L}) 22 \mathrm{Fl}-3 ; 35 \mathrm{E}$ \\
\hline$d p p^{H i n 37}$ & 1 & normal \\
\hline$D f(2 L) d p p^{H i n 38}$ & 2 & $\mathrm{Df}(2 \mathrm{~L}) 22 \mathrm{Al}-2 ; 22 \mathrm{~F} 3$ \\
\hline$D f(2 L) d p p^{\text {Hin } 39}$ & 2 & $\mathrm{Df}(2 \mathrm{~L}) 22 \mathrm{~A} 1-3 ; 23 \mathrm{~A} 2-4$ \\
\hline$D f(2 L) d p p^{H i n 40}$ & 2 & $\mathrm{Df}(2 \mathrm{~L}) 22 \mathrm{E} 1-2 ; 23 \mathrm{Al} 1-2$ \\
\hline$D f(2 L) d p p^{H i n 43}$ & 2 & $\begin{array}{l}\mathrm{Df}(2 \mathrm{~L}) 22 \mathrm{~A} ; 23 \mathrm{~A} 3-\mathrm{Bl}+ \\
\mathrm{T}(2 ; 3) 55 \mathrm{D}-\mathrm{E} ; 98 \mathrm{~F}\end{array}$ \\
\hline $\operatorname{In}(2 L R) d p p^{H i n 45}$ & 2 & $\begin{array}{l}\text { In(2LR) } 22 \mathrm{~F} 1-3 ; 22 \mathrm{C} \\
\text { superimposed upon } \\
\mathrm{Dp}(2 ; 2) \mathrm{dpp}^{\mathrm{d} 21}[\mathrm{Dp}(2 ; 2) \\
22 \mathrm{~A} 2,3 ; 22 \mathrm{~F} 1,2 \text { into } 52 \mathrm{~F}\end{array}$ \\
\hline$d p p^{\operatorname{Hin} 46}$ & 2 & normal \\
\hline$d p p^{\text {Hin } 47}$ & 2 & normal \\
\hline$d p p^{\text {Hin } 48}$ & 2 & normal \\
\hline$D f(2 L) d p p^{H i n 51}$ & 2 & $\operatorname{Df}(2 \mathrm{~L}) 21 \mathrm{~F} ; 23 \mathrm{Bl}-2$ \\
\hline$T(2 ; 3) d p p^{d 52}$ & 2 & $\mathrm{~T}(2 ; 3) 22 \mathrm{Fl}-2 ; 86 \mathrm{~A}-\mathrm{B}$ \\
\hline$D f(2 L) d p p^{H i n 53}$ & 2 & $\mathrm{Df}(2 \mathrm{~L}) 22 \mathrm{Al}-2 ; 23 \mathrm{~A} 3-7$ \\
\hline$d p p^{h i n-156}$ & 2 & normal \\
\hline$d p p^{H i n 57}$ & 2 & normal \\
\hline$d p p^{e 87}$ & 3 & normal \\
\hline$d p p^{\text {Hin88 }}$ & 3 & normal \\
\hline
\end{tabular}

a Details of mutagenesis protocols $1-3$ are described in Materials and methods. All $d p p$ lesions recovered in flies bearing a duplication of $d p p^{H i n+}$ material are listed.

ticular phenotypes arising from lesions in the $d p p^{\text {Hin }}$ region.

\section{The homozygous null phenotype}

By appropriate use of linked $d p p^{H i n+}$ duplications, animals that are functionally homozygous null can be generated (see Materials and methods). The null phenotype has been characterized in embryos bearing a $d p p^{H i n-}$ allele in trans to $D f(2 L) D T D 2$, a complete deficiency of the $d p p$ gene. The $d p p^{H i n-}$ alleles that have been so examined include $d p p^{H i n 37}, d p p^{H i n 46}, d p p^{H i n 47}, d p p^{H i n 48}$, and $d p p^{\text {Hin88. }}$. The phenotypes exhibited by all of these combinations are similar and cannot be distinguished from the phenotype of $D f(2 L) d p p^{H i n 38} / D f(2 L) D T D 2$ animals, which lack all $d p p$ sequences. Therefore, this phenotype represents the complete loss of zygotic $d p p^{H i n+}$ activity.

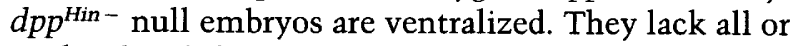
most head and thoracic cuticular structures and exhibit an almost complete replacement of the dorsal abdominal cuticle by ventral abdominal epidermal pattern elements. An example of the null phenotype is shown in Figure $2 \mathrm{H}$. In such animals, little or no cuticular material is present at the anterior end, resulting in a "hole" in mounted specimens. In favorable preparations, an occasional disarranged row of thoracic denticles is visible on the ventral surface just posterior to the hole. None of the cephalic sensory organs are present, including the mouth hooks, cirri, or the antenno-maxillary complexes. The internalized cephalo-pharyngeal skeleton and the thoracic Keilin's organs are absent in these animals. The first abdominal segment (Al) can be identified in wildtype embryos by its thinner ventral setal belt and by the posterior orientation of all the setae. These embryos frequently have a near normal Al cuticular morphology in that most of the characteristic rows of setae are present ventrally. However, the most anterior row of $\mathrm{Al}$ setae is often incomplete. The midabdominal segments, A2-A6, exhibit a variably ventralized phenotype. Generally about six sets of setal belts are distinguishable posterior to Al. These bands are not easily assigned to particular segments, since the characteristic shape and pattern of wild-type setal belts are absent. Instead, broader bands (encompassing as many as eight-ten rows of setae) are present and often completely encircle or spiral around the embryo. Normal dorsal-ventral polarity is lacking, and with the exception of rare examples containing a few scattered fine, dorsal-like setae, dorsal hairs are not observed. Furthermore, these embryos are often twisted within the vitelline membrane, and so the apparent dorsal surface as defined by the asymmetries of the eggcase may not correspond to the dorsal surface of the embryo. Posteriorly, disorganized rudiments of the dorsal spiracles and the Felzkörper are present internally. In some cases, no posterior sense organs are apparent, and the posterior end of the embryo is capped with a patch of setae which completely encircles the caudal tip.

Whole mounts of living embryos of the null genotype display aberrations in the first movements of gastrulation. As the germ band begins to extend, it moves into the interior of the embryo, rather than along the dorsal surface. In addition, the cephalic furrow also appears to invaginate more deeply than normal on the dorsal surface. This mutant phenotype becomes apparent $15 \mathrm{~min}$ after the onset of gastrulation (Fig. 3).

\section{Leaky dpp ${ }^{\text {hin-r }}$ phenotypes}

Animals trans-heterozygous for any two $d p p^{\text {hin-r }}$ alleles display normal dorsal-ventral polarity, but show defects in head and caudad formation (Figs. 2C-F). These dead embryos have uninvoluted heads, with most of the head-associated structures herniated antero-dorsally. The derivatives of the maxillary appendage, the cirri and the maxillary sense organs as well as the mouth hooks, are apparent and range laterally around the uninvoluted head material. The bulbous antennal sense organs can also be seen in favorable preparations (Fig. 2D). In the thoracic and abdominal regions, misfusions or gaps in the setal belts can occur, but all three thoracic and eight abdominal segments can be discerned unambiguously. The ventral thoracic sense organs, the Keilin's organs and the ventral pits, are detectable but often duplicated (Fig. 2E,F). In wild-type animals, Keilin's organs are composed of three hairs arising from a cluster of three cells. Struhl (1984) has suggested that the Keilin's organs straddle the compartment boundary, with two hairs derived from the anterior compartment and one hair from the posterior. In these recessive lethal combinations, frequently four and as many as five hairs arising from the Keilin's organ can be seen. Occasionally, the ventral 


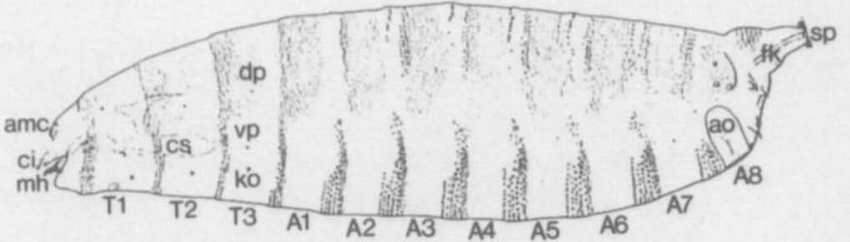

A
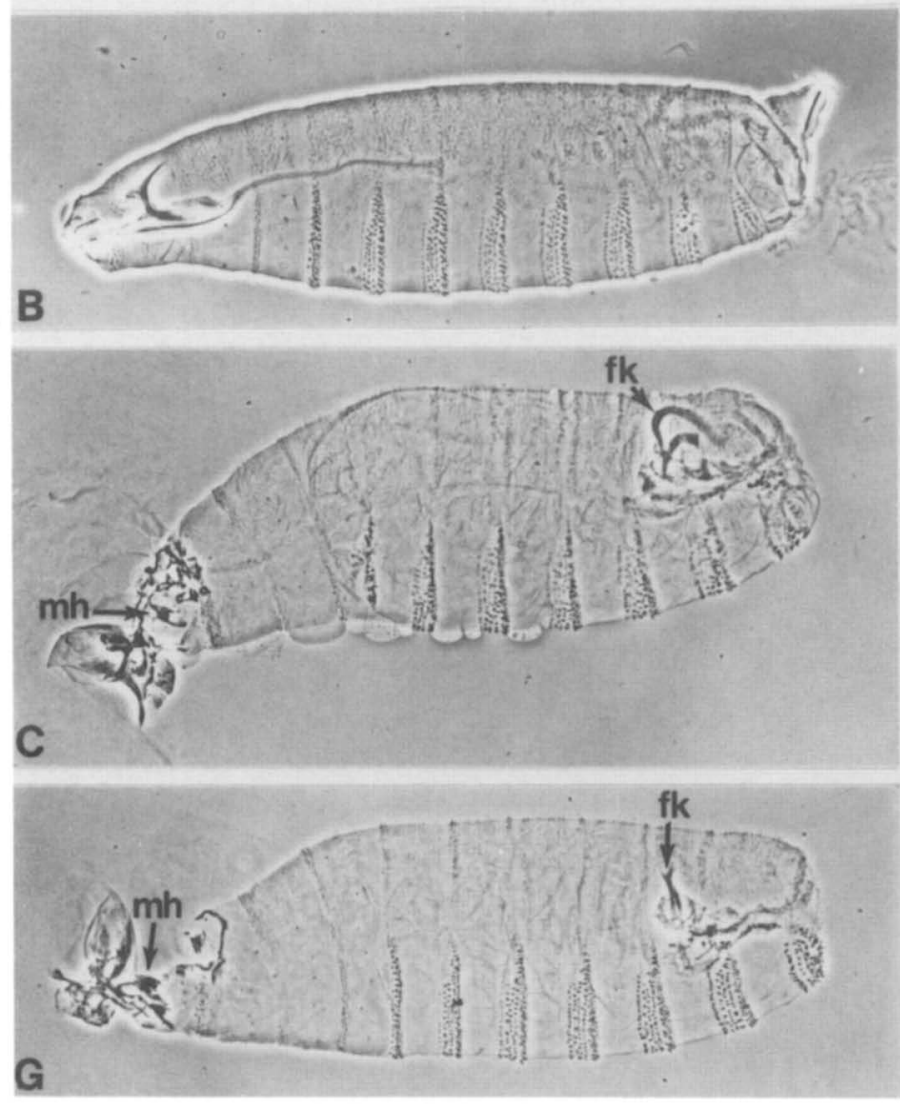

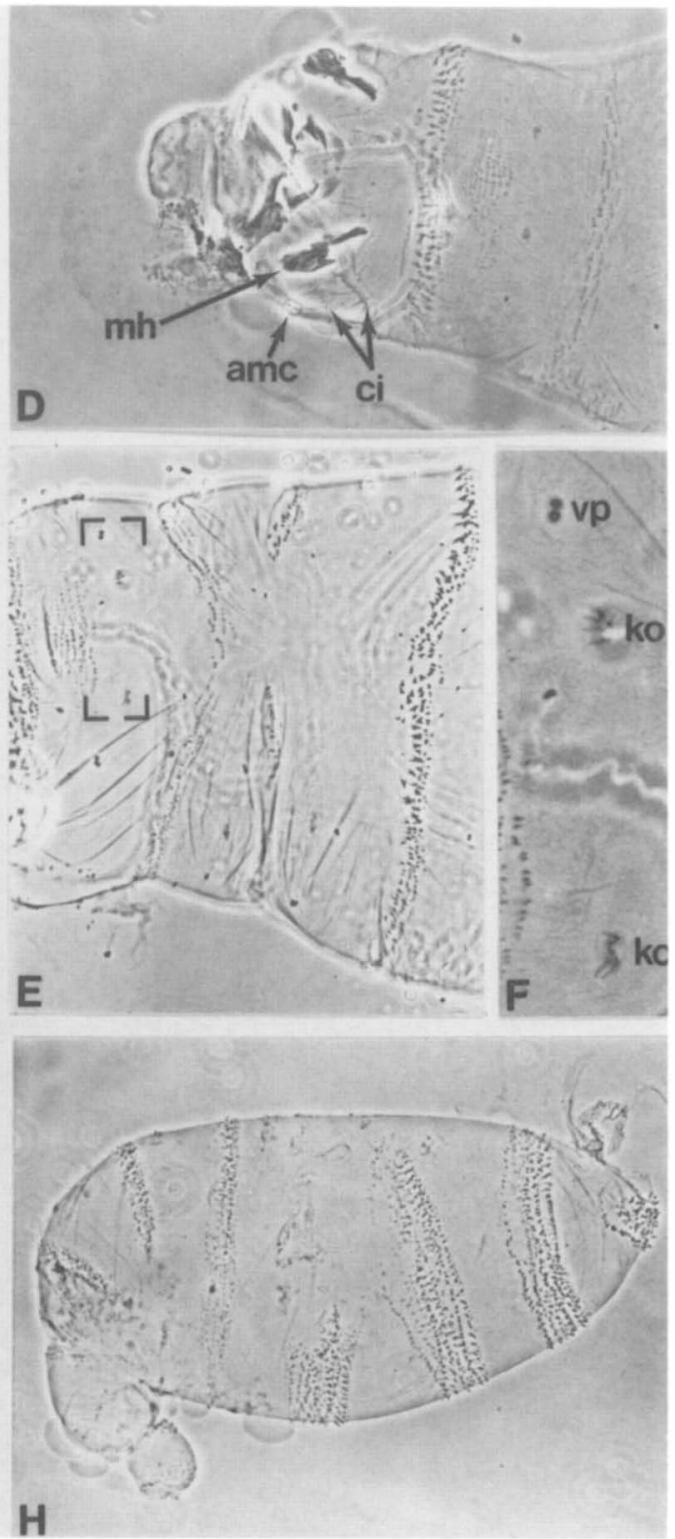

Figure 2. Cuticular phenotypes of $d p p$ embryonic lethal mutations. $|A|$ Diagram of a wild-type lateral view, anterior left, dorsal above (modified from Lohs-Schardin et al. 1979). Heavy stippling indicates segmental ventral setae; light stippling indicates dorsal hairs. $(B)$ Wild-type embryo, lateral phase contrast view. $(C) d p p^{\text {hin-r56/dpp }} p^{\text {e87 }}$ recessive lethal combination, lateral view. $(D) d p p^{\text {hin-556/ }}$ $d p p^{\text {hin-r89 }}$ head, ventral view. $d p p^{\text {hin-r89 }}$, formerly $s h v^{516}$, was initially identified as an allele of $s h v^{56}$ and subsequently shown to be a

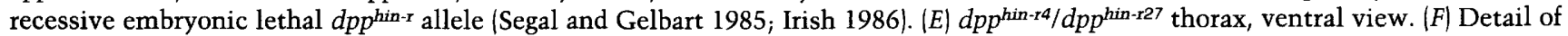
$E$. Note the duplicated Keilin's organs and ventral pits. $(G) D f(2 L) D T D 2$, dpp ${ }^{H i n-} /+$ haplo-lethal phenotype, lateral view. $(H)$ $d p p^{\text {Hin47 }} / D f(2 L) D T D 2, d p p^{\text {Hin - }}$ null phenotype, lateral view. (T1-T3) Thoracic segments 1-3; (Al-A8) abdominal segments 1-8; (amc) antenno-maxillary complex; (ao) anal organs; (cs) cephalopharyngeal skeleton; (ci) cirri; (dp) dorsal pits; (fk) Felzkörper; (ko) Keilin's organs; (mh) mouth hooks; (sp) posterior spiracles; (vp) ventral pits.

pits are duplicated immediately lateral to the normal location. Posteriorly, the spiracles and the associated Felzkörper are uneverted and can be seen internally. The spiracles often appear rudimentary. The uneverted material, which can include the eighth abdominal segment, runs along and just under the dorsal surface.

The dominant haplo-lethal $d p p^{\text {Hin- } / d p p^{H i n+} \text { cuticular }}$ phenotype largely overlaps that of recessive embryonic lethal $d p p$ combinations (cf. Figs. 2C,G). This similarity supports our proposal that the recessive lethal alleles are leaky for the $d p p^{H i n}$ product. Animals either monosomic for the $d p p$ gene, or heterozygous for a $d p p^{H i n-}$ and a wild-type allele were scored for their cuticular phenotypes. For instance, $D f(2 L) D T D 2, d p p^{H i n-} / d p p^{+}$animals show this characteristic embryonic lethal phenotype (Fig. 2G). These embryos have normal dorsal-ventral 


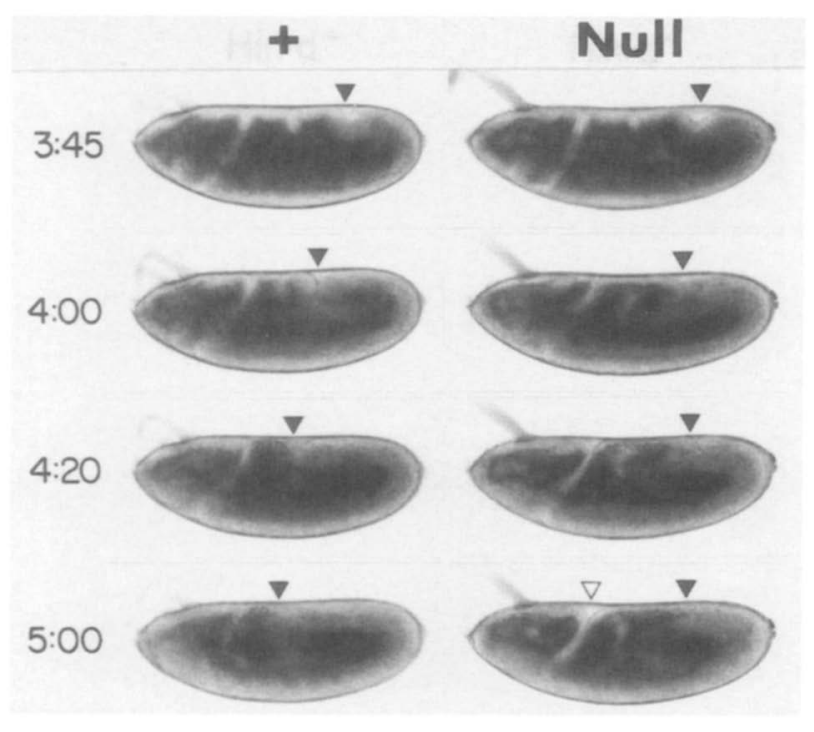

Figure 3. Gastrulation phenotype of $d p p^{\text {Hin }-}$ null embryos. (Left and right) Time-lapse photographs of developing wild-type

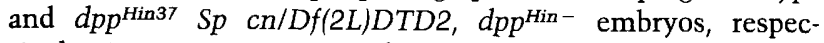
tively. Putative mutant embryos, resulting from a cross of $D f(2 L) D T D 2, d p p^{\text {Hin- }} / D p(2 ; 2) M V D 1-D 2$ females and $d p p^{H i n 37 /}$ $D p(2 ; 2) D T D 48$ males, were followed until their genotypes could be confirmed by examining their cuticular phenotypes. Anterior is to the left and the dorsal side is above. Numbers indicate time (in hours and minutes) after fertilization. Closed triangles indicate the extent of germ band elongation as marked by the forward movement of pole cells. The open triangle indicates the abnormally deep dorsal cephalic furrow in the $d p p^{\text {Hin - }}$ embryo. Note that the only abnormal embryos produced by this cross are null for $d p p$. Functionally, both copies of $d p p$ on the $D p(2 ; 2) M V D 1-D 2$ chromosome behave as $d p p^{H i n+}$ with regard to the dominant lethal $d p p$ phenotype (see Materials and methods for the exact genotype). These copies are recombinationally inseparable from one another. Similarly, the two copies of $d p p$ on the $D p(2 ; 2) D T D 48$ chromosome are functionally $d p p^{\text {Hin + }}$, and because this chromosome is introduced patroclinously into the offspring, the two $d p p^{H i n+}$ copies are completely linked. Thus, the only zygotes deriving from this cross are either null, disomic, or tetrasomic for $d p p^{\mathrm{Hin}+}$; as confirmed by egg-counting experiments, all disomic and tetrasomic offspring survive to adulthood.

polarity, but are defective in head and caudad formation; they also display duplications of the ventral thoracic sense organs.

The $\mathrm{dpp}^{\mathrm{Hin}}$ region does not encode any maternally acting components

The mutant embryonic phenotypes associated with $d p p^{H i n}$ and $d p p^{\text {hin-x }}$ lesions demonstrate that the $d p p$ gene is required early in development. To test the possibility that maternal $d p p^{H i n}$ expression may also contribute to normal embryonic development, we examined the phenotypes of embryos derived from homozygous mutant oogonia. Clones of homozygous mutant cells can be produced by mitotic recombination in the female germ line. Such clones can be recognized by placing the mutation to be studied in trans to a dominant female sterile mutation (Wieschaus et al. 1981). Only the ovarian tissues in which a homozygous mutant clone has been induced will be able to support oogenesis. This technique was utilized to identify homozygous $d p p^{\text {Hin- }}$ germ line clones. Because $d p p^{\mathrm{Hin}-}$ mutations themselves are dominant lethal, an allele with a leaky dominant phenotype, $d p p^{H i n 88}$, was chosen for analysis. $d p p^{H i n 88} / d p p^{+}$heterozygotes frequently survive to adulthood, but $d p p^{H i n 88 /}$ $D f(2 L) d p p^{H i n-}$ animals do have a strongly ventralized phenotype indistinguishable from the null state. Thus, in a null background, $d p p^{H i n 88}$ eliminates all $d p p^{\text {Hin }}$ function. Clones of germ line tissue homozygous for $d p p^{H i n 88}$ were produced by inducing mitotic recombination in $d p p^{\text {Hin88 }} F s(2) 1^{+} / d p p^{+} F s(2) 1$ second-instar larvae and screening the resulting escaper females for egg production. $F s(2) 1$ is a dominant germ line-autonomous female sterile mutation that eliminates egg production (kindly provided by J. Szabad). Only females carrying $d p p^{\text {Hin88/ }} d p p^{\text {Hin88 }}$ germ line tissue, resulting from an appropriate mitotic recombination event, will overcome the sterility of $F s(2) 1$ and have the potential to produce oocytes (Fig. 4).

Three types of crosses were performed to examine the phenotypes of embryos derived from homozygous $d p p^{H i n 88}$ germ line tissue. The crosses differed in paternal genotypes to allow for the production of zygotes bearing 0,1 , or 2 copies of $d p p^{H i n+}$ from homozygous $d p p^{\text {Hin88 }}$ oogonia.

In crosses of irradiated escaper females to homozygous $D p(2 ; 2) D T D 48, d p p^{d-h o}$ males, five females bearing clones were recovered (Table 2, cross 1). All resulting zygotes have two copies of $d p p^{\mathrm{Hin}+}$ and therefore should be viable based on their zygotic genotype, but are derived from oogonia deficient for $\mathrm{dpp}^{\text {Hin }}$ product. The $90 \%$ survival to adulthood of these zygotes indicates either that there is no maternal requirement for $d p p^{\text {Hin }}$ activity or that such a requirement can be zygotically rescued. These surviving adults appeared phenotypically normal and were fertile.

Crosses of irradiated escaper females to $d p p^{\mathrm{Hin}+}$ males were designed to assess whether loss of maternal $d p p^{\mathrm{Hin}+}$ product had an effect on the phenotype of animals containing one wild-type dose of $d p p^{\text {Hin }}+$ $\left(d p p^{H i n 88} / d p p^{H i n+}\right)$. In these crosses, all progeny should be $d p p^{\text {Hin88 }} / d p p^{\text {Hin }+} ; 42 \%$ were escapers surviving past hatching (Table 2, cross 2). This high escaper frequency is consistent with that obtained in crosses of $d p p^{\text {Hin88 }}$ involving $d p p^{H i n+}$ mothers (Irish 1986). The dead embryos recovered from this cross show a range of phenotypes indistinguishable from those of $d p p^{\mathrm{Hin}-} / d p p^{\mathrm{Hin}+}$ animals described in a previous section.

Several crosses were designed to examine the phenotypes of $d p p^{H i n 88} / D f(2 L) D T D 2$ embryos derived from homozygous $d p p^{H i n 88}$ oogonia. Escaper females were mated to either $D f(2 L) D T D 2 / d p p^{\text {hin-r4 }} D p(2 ; 2) M V D 1-D 2, d p p^{d-}$ ho males or $D f(2 L) D T D 2 / I n(2 L) d p p^{d 10} D p(2 ; 2) D T D 48$, $d p p^{d-h o}$ males. Half of the resulting zygotes from these crosses should lack $d p p^{H i n+}$ completely, whereas the other half should carry two copies of $d p p^{H i n+}$. Four clones were recovered from the $d p p^{H i n 88} / F s(2) 1$ females mated to $D f(2 L) D T D 2 / d p p^{h i n-14} D p(2 ; 2) M V D 1-D 2, d p p^{d-}$ ${ }^{h o}$ males. Of the progeny derived from these clones, $53 \%$ 


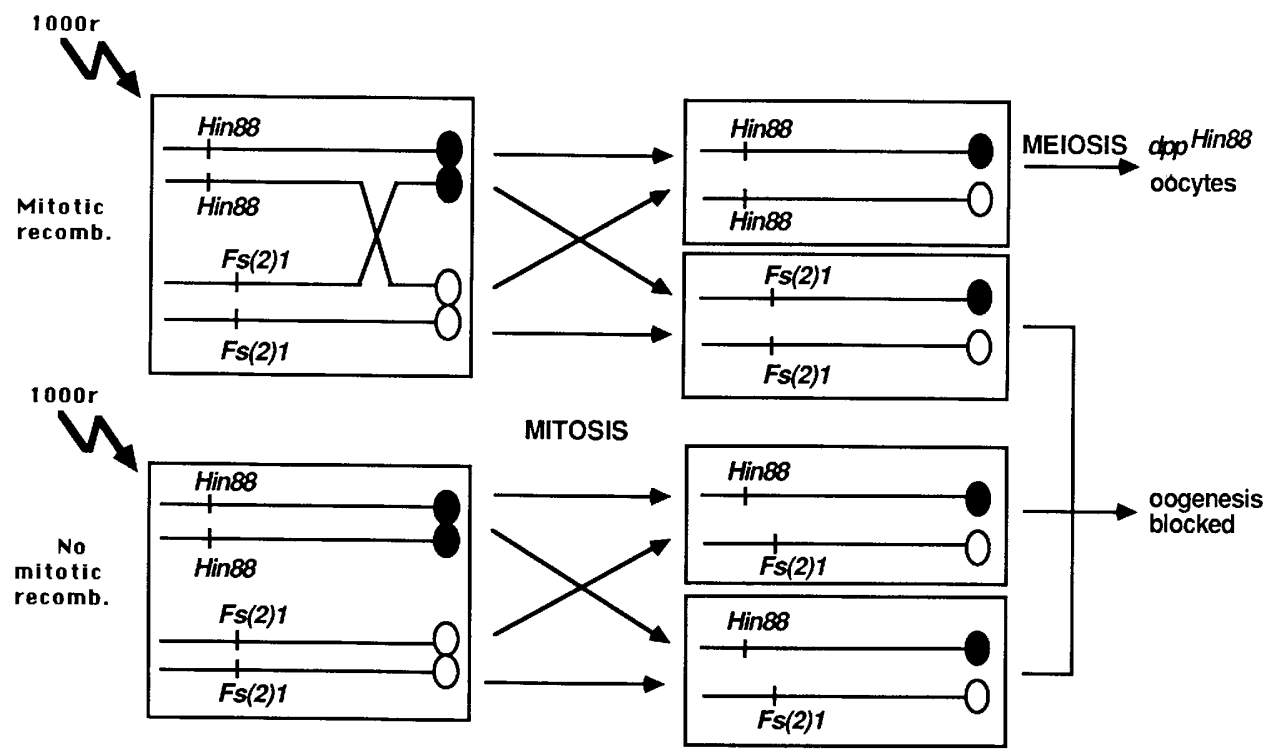

Figure 4. Generation of germ line clones by mitotic recombination. The result of inducing a mitotic recombination event in a germ-line stem cell is diagrammed. The first panel illustrates the genotype of stem cells at $\mathrm{G}_{2}$ of mitosis. For simplicity, only chromosome arm $2 \mathrm{~L}$ is shown. A proximal mitotic recombination event on chromosome arm $2 \mathrm{~L}$ and subsequent mitosis will generate two populations of premeiotic germ cells, here shown in $\mathrm{G}_{1}$. In the absence of mitotic recombination, heterozygous premeiotic germ cells will result. Cells either homozygous or heterozygous for Fs(2)1 will not produce any eggs. Only those cells homozygous for $d p p^{\text {Hin88 }}$ will be capable of producing oocytes.

died during embryogenesis (Table 2, cross 3). Five clones were found in similar crosses of $d p p^{\text {Hins8 }} / F_{S}(2) 1$ escaper females to $D f(2 L) D T D 2 / \operatorname{In}(2 L) d p p^{d 10} \quad D p(2 ; 2) D T D 48$, $d p p^{d \cdot h o}$ males. Of the progeny of this cross, $57 \%$ died during the embryonic period (Table 2, cross 4). The dead embryos recovered from these crosses (Fig. 5) have a phenotype indistinguishable from the null phenotype described earlier (Fig. 2H). These animals appear ventralized, with much of the head and thoracic material missing or disorganized. About six abdominal ventral setal belts are apparent and usually continue around the body of the animal. Posteriorly, rudimentary Felzkörper material and often a patch of ventral denticles are internalized. Anteriorly, none of the cephalic structures can be detected, but an occasional row of thoracic denticles can be seen. The results of these crosses indicate that the absence of maternal $d p p^{\mathrm{Hin}+}$ product does not affect the zygotic phenotype.
The epistatic relationships between dorsalizing maternal-effect mutations and $\mathrm{dpp}^{\mathrm{Hin}}$ alleles

The ventralized phenotype associated with $d p p^{\mathrm{Hin}-}$ null embryos demonstrates that $d p p^{H i n}$ zygotic activity is required for normal dorsal-ventral pattern formation in the embryo. The dorsal-ventral pattern is initially defined by a set of maternally acting genes, including dorsal (dl), pelle (pll) and Toll (Tl) (Anderson and Nüsslein-Volhard 1984a,b). By analyzing the effects of removing both the dorsalizing gene product in the mother and the $d p p^{H i n}$ product in the zygote, we hoped to identify the relationships between these genes involved in dorsal-ventral patterning.

Zygotes entirely lacking $d p p$ were derived from mothers homozygous for $T 1^{\text {r632-5 }}$, $p l 1^{385-13}$, or $d l^{1}$. $d p p^{\text {Hin }}+$ embryos derived from $\mathrm{pll}^{385-13}$ or $d l^{1}$ mothers will have a dorsalized phenotype, consisting of dorsal setae covering the entire body, with rudimentary posterior spiracles

Table 2. Analysis of germ-line clones induced in $\mathrm{dpp}$ Hin88 $\mathrm{dp} \mathrm{cn}$ bw/Fs $(2) 1$ females

\begin{tabular}{|c|c|c|c|c|c|c|}
\hline Cross & Male genotype & $\begin{array}{l}\text { Females } \\
\text { with clones }\end{array}$ & $\begin{array}{l}\text { Number of } \\
\text { embryos examined }\end{array}$ & $\begin{array}{l}\text { Number of } \\
\text { dead embryos }\end{array}$ & $\begin{array}{l}\text { Number of } \\
\text { hatched }\end{array}$ & $\begin{array}{l}\text { Number of } \\
\text { adults }^{\mathrm{a}}\end{array}$ \\
\hline 1 & $d p p^{+} D p(2 ; 2) D T D 48, d p p^{d \cdot h o}$ & 5 & 93 & nd & nd & 84 \\
\hline 2 & $d p p^{+} d p c n b w$ & 2 & 69 & 40 & 29 & 21 \\
\hline 3 & $\begin{array}{l}D f(2 L) D T D 2, d p p^{H i n-/} \\
\quad d p p^{\text {hin-r4 }} D p(2 ; 2) M V D 1-D 2, d p p^{d-h o}\end{array}$ & 4 & 148 & 79 & 69 & 61 \\
\hline 4 & $\begin{array}{l}D f(2 L) D T D 2, d p p^{H i n-}- \\
\quad \operatorname{In}(2 L) d p p^{d 10} D p(2 ; 2) D T D 48, d p p^{d \cdot h o}\end{array}$ & 5 & 152 & 86 & 66 & 64 \\
\hline
\end{tabular}

(nd) Not determined.

a All adults recovered in crosses 1,2 , and 3 carried $d p p^{\text {Hin88 }}$ and $d p$, as ascertained by test crosses to appropriately marked strains. In cross 4 , all adults recovered had a mild dpp ${ }^{\text {disk }}$ phenotype, indicative of the presence of the maternally derived $d p p^{\text {Hin } 88}$ mutation. 


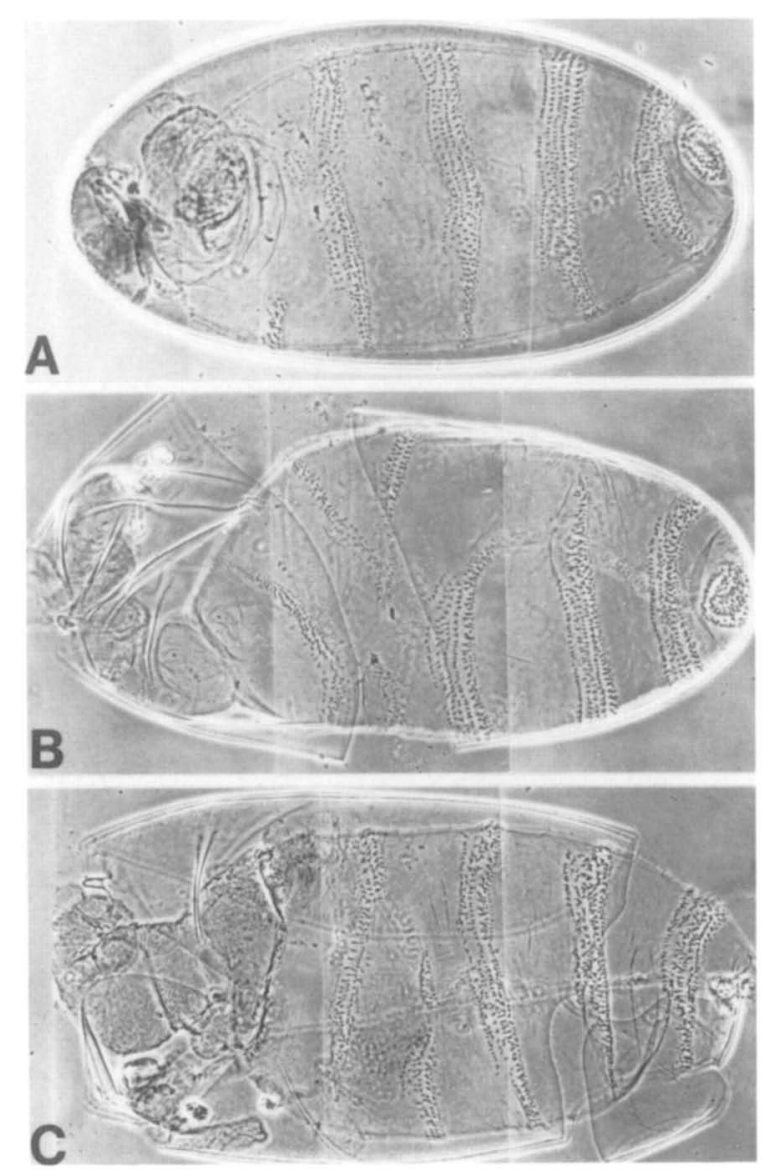

Figure 5. $d p p^{\text {Hin }}$ null embryos from homozygous null germ line clones. Phase contrast views of three embryos derived from maternal germ lines homozygous for $d p p^{H i n 88}$. Anterior is to the left and dorsal is above. $A$ and $B$ are derived from one clone, whereas $C$ is derived from another. All three embryos were recovered in cross 4 (see Table 2).

lacking Felzkörper. In addition, these animals have a rather elongated and convoluted appearance (e.g., see the dorsalized embryo depicted in Fig. $6 \mathrm{~A}) . d p p^{\mathrm{Hin}+}$ animals derived from $T 7^{\mathrm{r} 632-5}$ mothers have a slightly weaker dorsalized phenotype than those derived from $p l^{1385-13}$, or $d l^{1}$ mothers (Anderson et al. 1985b and our observations). In all of these crosses, a class of embryos were recovered that had numerous ventral-like setae. As this phenotype is never seen in control embryos derived from homozygous $T 1^{1632-5}$, $\mathrm{pll}^{385-13}$ or $d l^{1}$ mothers, we conclude that these embryos are genotypically null for $d p p$. These doubly mutant animals display ventral-like setae that are of a size suggesting that they derive from the lateral portions of the ventral setal belts (Fig. 6B-D). These lateralized structures are somewhat disorganized and grouped into patches or belts that encircle the embryo. These animals do not have recognizable head structures, posterior spiracles, or Felzkörper. Whereas the most extremely ventralized embryos from the $T 1^{r 632-5}$ mothers had a similar phenotype to $d p p^{\text {Hin }-}$ embryos derived from $\mathrm{Tl}^{+}$mothers, the remaining putative $d p p^{\mathrm{Hin}-} \mathrm{em}-$ bryos from the $T 1^{-}$mothers, as well as those derived from $d l^{1}$ and $p l^{385-13}$ mothers, appear to be lateralized. The appearance of strongly ventralized $d p p^{H i n-}$ animals in the $T 7^{1632-5}$ cross may be due to the somewhat weaker phenotypic effects of the $T^{\text {r632-5 }}$ allele. Though extensive setal belts are seen in embryos from all three crosses, they still retain the convoluted tube phenotype characteristic of dorsalized embryos. These features, the relatively small-sized ventral setae and the convoluted tube phenotype, are characteristic of a lateralized phenotype (K. Anderson, pers. comm.).

\section{Interactions of $\mathrm{dpp}^{\mathrm{Hin}}$ mutations with other $\mathrm{dpp}$ lesions}

Dominant haplo-lethal mutations cannot be readily assayed for allelic interactions with other lesions, since the dominant lethality will preclude the observation of adult phenotypes. Two methods have allowed us to circumvent this problem. Occasional $d p p^{\mathrm{Hin}-/} d p p^{\mathrm{Hin}+}$ adult escapers can be recovered. To characterize interactions with other dpp mutations, male flies of the general genotype $D p(2 ; 1) G 146, d p p^{+} / Y$; $d p p^{H i n-} / \operatorname{In}(2 L R) C y O$, $C y$ were crossed to females bearing either a $d p p^{d i s k}$ or a $d p p^{s h v}$ lesion. The rare escapers from haplo-lethality were recovered among the progeny, and the escapers' phenotypes could then be characterized. Alternatively, flies of the general genotype $d p p^{H i n-} D p(2 ; 2) d p p^{d 21 /}$ In $(2 L R) C y O, C y$ were crossed to flies bearing a dpp disk mutation. Since $D p(2 ; 2) d p p^{d 21}$ is $d p p^{s h v+}$ and $d p p^{\text {Hin }+}$ but does not contain any functional sequences from the $d p p^{d i s k}$ region (Segal and Gelbart 1985), the progeny resulting from such a cross can be scored for their dpp disk phenotypes.

The phenotypes exhibited by $d p p^{H i n-} / d p p^{d i s k}$ combinations demonstrate that $d p p^{\mathrm{Hin}-}$ haplo-lethal mutations inactivate all $d p p^{d i s k}$ functions. Flies bearing $d p p^{\text {Hin37 }}$ or $d p p^{H i n 45}$ in trans to a $d p p^{d i s k}$ allele display the mutant phenotype associated with that $d p p^{d i s k}$ class. These two $d p p^{H i n}$ mutations are associated with rearrangements (Table 1 and Gelbart et al. 1985) and appear to eliminate all $d p p$ activity genetically. The pseudopoint lesions $d p p^{\mathrm{Hin} 46}, d p p^{\mathrm{Hin} 47}$, and $d p p^{\mathrm{Hin} 48}$ reduce or eliminate $d p p$ activity but are sensitive to transvection effects. When synapsis is prevented by rearrangement heterozygosity, these mutant phenotypes approximate the severity of those elicited by null $d p p^{\text {Hin }}$ alleles. The few escapers of $d p p^{\text {Hin }-/} / d p p^{\text {shv }}$ genotypes all display extreme wing venation defects, demonstrating that these $d p p^{H i n}$ lesions inactivate $d p p^{\text {shv }}$ functions as well.

Some recessive embryonic lethal $d p p$ mutations also partially inactivate $d p p^{d i s k}$ and $d p p^{\text {shv }}$ functions. The interactions displayed by $d p p^{\text {hin-54 }}$ and $d p p^{\text {hin-r27 }}$ in combination with $d p p^{d i s k}$ and $d p p^{s h v}$ alleles have been described in detail previously (Spencer et al. 1982; Segal and Gelbart 1985). $d p p^{\text {hin.r56 }}$ behaves in a similar fashion

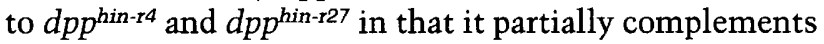
$d p p^{d i s k}$ and $d p p^{s h v}$ functions. However, $d p p^{e 87}$, which was initially recovered as a lethal allele of a $d p p^{\text {hin-r }}$ tester, is unique in that it complements all $d p p^{d i s k}$ and $d p p^{s h v}$ lesions. Therefore, it is likely that $d p p^{e 87}$ eliminates a function required solely during embryogenesis. 
Figure 6. Examples of $d p p^{H i n+}$ and $d p p^{H i n-}$ embryos derived from homozygous $d l^{1}$ mothers. Phase contrast views of embryos derived from $d l^{1}$ homozygous mothers. (A) An example of a dorsalized $d p p^{H i n+}$ embryo removed from its vitelline membrane. Note the absence of ventral setae. $(B, C)$ Two focal planes through a presumed $d p p^{H i n-}$ lateralized embryo derived from a cross in which both $d p p^{H i n+}$ and $d p p^{H i n-}$ embryos were generated. This embryo still has a tube-like conformation characteristic of dorsalized embryos, but it has several bands of lateralized setae never found in $d p p^{H i n+}$ individuals derived from $d l^{1}$ mothers. Some of the setae outlined in $C$ are shown at higher magnification in $D$. (sp) Posterior spiracle.

\section{Discussion}

The embryonic effects of dpp mutations

The phenotypes of embryos mutant for $d p p^{H i n}$ fall into several categories. The $d p p^{H i n}$ null phenotype consists of an almost complete ventralization of the embryo. These animals lack dorsal cuticular structures, in addition to showing extreme cephalic and caudal defects. Because $d p p^{H i n-} / D f(2 L) d p p^{H i n-}$ animals heterozygous for a $d p p^{\text {Hin }}$ - pseudo-point mutation display a ventralized phenotype indistinguishable from that of a $D f(2 L) d p p^{H i n}$ homozygote, the $d p p^{\text {Hin - }}$ pseudo-point mutations can be considered amorphic or null lesions.

The $d p p^{H i n-}$ null phenotype is novel among zygotically expressed genes. Whereas mutations at several other loci have been reported to cause ventralization effects, they are all distinguishable from the $d p p^{H i n}$ null phenotype either in time of expression (zygotic vs. maternal) or in the severity of the null phenotype. Embryos from mothers bearing gain-of-function Toll-Dominant mutations appear ventralized and lack structures derived from the dorsal and dorsolateral anlagen, including head sensory organs and posterior structures (CamposOrtega 1983; Anderson et al. 1985a,b). In contrast to the dorsalized embryos derived from homozygous Toll-recessive mothers, Toll-Dominant-derived animals gastrulate abnormally but maintain normal dorsoventral polarity (Anderson et al. 1985b). The $d p p^{\text {Hin }}$ null zygotic phenotype is similar to the Toll-Dominant maternal-effect phenotype in the extent of ventralization and the retention of normal polarity during gastrulation. The only other zygotic lethal loci resulting in a ventralizing phenotype are zen, tolloid, and shrew (Wakimoto et al. 1984; Jurgens et al. 1984; Frohnhofer 1982); these loci, when mutated, produce much less severely ventralized cuticle. Thus, loss-of-function mutations of $d p p^{H i n}$ result in the most severely ventralized phenotype known among genes expressed exclusively in the zygote.

As null $d p p^{\text {Hin }}$ mutations are dominant loss-of-function lethals, previous screens for embryonic pattern mutations would not have identified such haplo-lethal lesions. In fact, only one weak recessive $d p p$ allele has been isolated in a general screen for second-chromosome embryonic lethal mutations (Nüsslein-Volhard et al. 1984). Possibly, strongly ventralizing mutations in other loci can arise but are not recovered due to similar haplolethal effects.

The $d p p^{\text {Hin }}$ null phenotype could result from cell

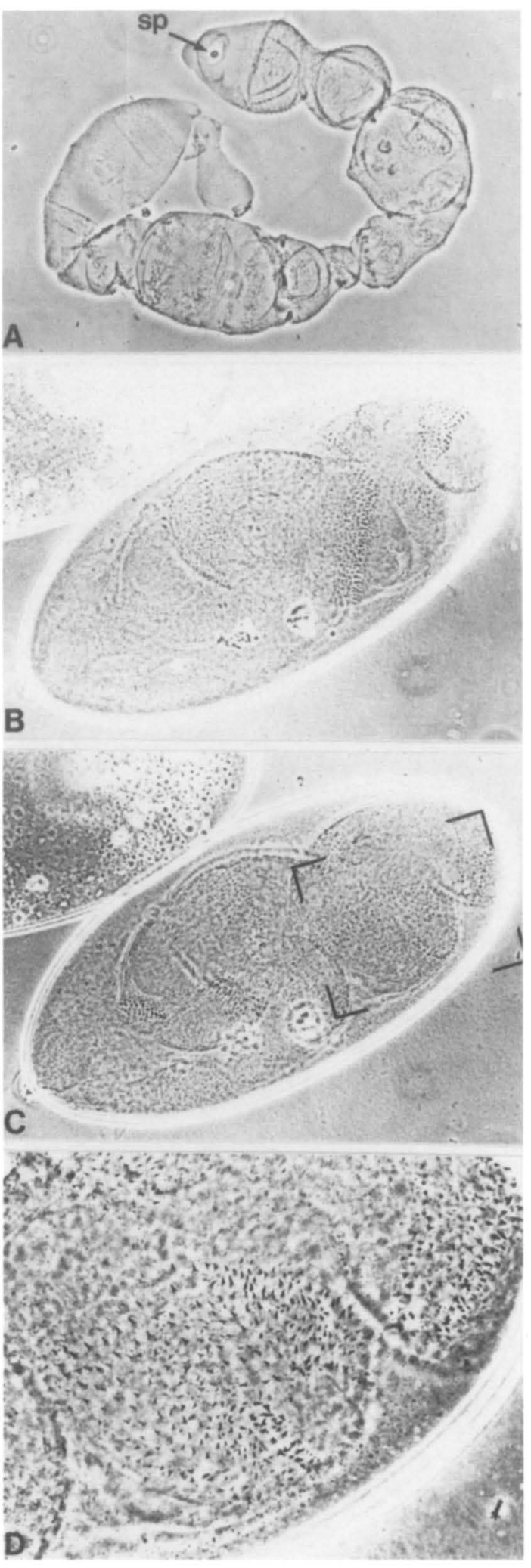

death of dorsal epidermis followed by overgrowth of ventral tissue. Alternatively, this phenotype could arise by the transformation of dorsal epidermis into ventral epidermis. Homozygous $d p p^{H i n-}$ zygotes generated from homozygous $T l^{r 632-5}$, $p 11^{385-13}$, or $d l^{1}$ mothers result in lat- 
eralized embryos. The effect of removing both the dorsalizing maternal and $d p p^{\mathrm{Hin}}$ zygotic gene products shows that $d p p^{H i n+}$ is required for the expression of the dorsalized phenotype. Minimally, we can conclude that the lack of $d p p^{H i n+}$ function overcomes the absence of $T 1^{+}$, $\mathrm{pll}^{+}$, and $\mathrm{dl}^{+}$products to direct the development of some ventral epidermal structures. This supports the idea that in embryos from wild-type mothers, $d p p^{\mathrm{Hin}-}$ mutations cause the transformation of dorsal into ventral epidermis. If tissue determined to become dorsal epidermis dies in the $d p p^{H i n-}$ null embryos, then one should not see ventral or lateral tissue formed in the double-mutant combination. Rather, no epidermis should be formed in these animals. The appearance of lateral tissue in the double-mutant combination supports a model in which $d p p^{H i n-}$ mutations result primarily in transformation of cell fate, not in cell death. Thus, we think it likely that $d p p^{\mathrm{Hin}+}$ expression plays a determinative role in embryonic dorsal tissue development.

The $\mathrm{Tl}^{+}$gene is thought to encode a substance required for the formation of a chemical reaction-diffusion system (Anderson et al. 1985a,b). The $\mathrm{dl}^{+}$product may interact with the $\mathrm{Tl}^{+}$gene product to create a dorsal-ventral morphogen gradient (Anderson et al. 1985b). The high point of this putative morphogen gradient is located ventrally. Given this idea of a dorsalventral gradient, we have developed the working hypothesis that, directly or indirectly, genes of the dorsalizing class such as Toll act as negative regulators of $d p p^{H i n}$ expression, thereby determining a part of the dorsal-ventral pattern of the epidermis. The dorsalized epidermal phenotypes of $d p p^{H i n+}$ embryos derived from mothers homozygous for $T 7^{\mathrm{r} 632-5}$, $p 11^{385-13}$, or $d 1^{1}$ would then be explained as due to the indiscriminate expression of $d p p^{\text {Hin }}$ product throughout the dorsal and ventral epidermis and perhaps throughout all the germ layers. The lateralized phenotype of $d p p^{H i n-}$ embryos derived from $\mathrm{Tl}^{-}, \mathrm{pll}^{-}$, or $\mathrm{dl}^{-}$mothers demonstrates that $d p p^{\text {Hin }}$ is not completely epistatic to the dorsalizing maternaleffect genes tested. It is likely that this phenotype is due to the aberrant regulation and expression of other $z y$ gotic genes involved in dorsal-ventral patterning. We suggest that $d p p^{H i n}$ expression may be a major zygotic determinant for dorsal versus lateral levels of epidermis.

Both genetic and transplantation experiments are beginning to define how cells along the dorsal-ventral axis acquire a specific identity. Fate mapping experiments (Campos-Ortega and Hartenstein 1985) have defined four dorsal-ventral regions within the segmented portion of the embryo: the mesoderm, the ventral neurogenic region, the dorsal epidermis, and the amnio-serosa. Cells within at least the dorsal epidermal region, however, are still pluripotent at the onset of gastrulation, and upon transplantation can differentiate into derivatives of the ventral neurogenic region (Technau and Campos-Ortega 1986). These results imply that commitment of gastrula cells to a dorsal epidermal fate is not lineage dependent, but instead relies on the acquisition of specific positional cues. Given the recent finding that cDNAs isolated from the $d p p^{H i n}$ region encode a se- quence homologous to proteins of the mammalian transforming growth factor- $\beta$ family (Padgett et al. 1987), it is likely that $d p p^{\text {Hin }}$ product conveys positional information by acting at the level of intercellular communication.

A growth factor-like protein encoded by $d p p^{H i n}$ may define position along the dorsal-ventral axis in one of two ways. $d p p^{H i n}$ activity could be turned on solely in the presumptive dorsal epidermis and act only in those cells to define that region. The lateralized phenotypes seen in the $d p p^{H i n-}$ embryos derived from $\mathrm{Tl}^{-}, \mathrm{pll}^{-}$, and $\mathrm{dl}^{-}$mothers suggests that in the absence of this information, other genes responsible for ventro-lateral differentiation are activated and produce this cuticular phenotype. However, other evidence supports the idea that $d p p^{H i n}$ may define a zygotic gradient within the whole epidermal field. The weakly ventralized phenotype displayed by $d p p^{h i n-r} / d p p^{\text {hin-r }}$ or $d p p^{\text {Hin- }} / d p p^{\text {Hin }+}$ animals, notably the laterally duplicated ventral pits and Keilin's organs, suggests that a reduction in wild-type $d p p^{H i n}$ activity expands the ventro-lateral epidermal anlage. The sensitivity of the fate of dorsal epidermal cells to their local environment (Technau and Campos-Ortega 1986) could be viewed as consistent with this latter possibility of a gradient of $d p p^{H i n}$ product. To distinguish these alternative proposals for $d p p^{H i n}$ function, it will be necessary to ascertain if the $d p p^{\text {Hin }}$ product acts in a localized or in a nonautonomous manner to determine cell fate in the embryonic epidermal anlagen.

Organizational aspects of $\mathrm{dpp}$

We appear to have identified alleles affecting embryonic function which are either null or have low levels of $d p p$ expression. The idea that the recessive embryonic lethal alleles are leaky $d p p^{\text {Hin }}$ mutations is consistent with observations of interactions with mutations elsewhere in dpp. trans combinations of recessive embryonic lethal or haplo-lethal $d p p^{H i n}$ alleles with either $d p p^{d i s k}$ or $d p p^{\text {shv }}$ lesions elicit a variety of phenotypes. In heterozygotes with a given $d p p^{d i s k}$ or $d p p^{s h v}$ allele, the haplo-lethal $d p p^{H i n}$ alleles tend to exhibit more extreme mutant phenotypes than do the recessive embryonic lethal $d p p^{\text {hin-r }}$ alleles. Insofar as we have been able to test them, the haplo-lethal $d p p^{\mathrm{Hin}}$ alleles behave phenotypically as if they completely inactivate all functions of the complex. In contrast, the recessive embryonic lethal $d p p^{\text {hin-r }}$ alleles appear to retain some residual activity for all $d p p$ functions.

We have previously proposed that the $d p p^{H i n}$ region encodes a protein product as well as regulatory signals necessary for normal embryonic development, with the $d p p^{s h v}$ and $d p p^{d i s k}$ regions consisting of vast arrays of $c i s-$ acting regulatory elements that control expression of the $d p p^{\text {Hin }}$ product(s) necessary for later developmental stages (Gelbart et al. 1985). Such a model, in which $d p p^{d i s k}$ regulatory elements control the expression of $d p p^{H i n}$-encoded functions accounts for the phenotypic interactions elicited in $d p p^{\text {Hin }-/ d p p^{d i s k}}$ genotypes. Furthermore, such a model would require that at least some functions of the $d p p^{H i n}$ region are necessary for imaginal disk development. In this regard, $d p p^{e 87}$, the one reces- 
sive embryonic lethal allele that complements both $d p p^{d i s k}$ and $d p p^{s h v}$ lesions, is particularly intriguing. It is associated with a small deletion in or near the $d p p^{H i n}$ region (Irish 1986; R.D. St. Johnston and W.M. Gelbart, unpubl.), and is our only mutation that defines a function required solely during embryogenesis. Thus, $d p p^{e 87}$ may be the first example of a $d p p$ mutation specifically impairing a regulatory element required for normal embryonic expression. Given that we now know such lesions can be generated, a global view of the structure and regulation of $d p p$ will require the identification and characterization of a number of similar stage-specific mutations.

\section{Materials and methods}

\section{Strains and nomenclature}

Since recent molecular analyses (Gelbart et al. 1985; Padgett et al. 1987; R.D. St. Johnson, R.W. Padgett, H.A. Irick, S.D. Findley, and W.M. Gelbart, unpubl.) indicate that $d p p$ encodes a single polypeptide, we feel that it is not appropriate to refer to a "decapentaplegic gene complex," but rather to treat decapentaplegic as a single gene with complex allelic interactions. We have revised our nomenclature accordingly. In our new system, all decapentaplegic mutations are referred to as $d p p$ alleles. The $d p p$ gene is divided into three regions, which have been re-

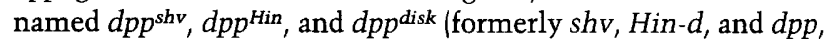
respectively). $d p p^{s h v}$ region mutations, formerly $s h v^{s x}$, are now named $d p p^{s x} . d p p^{H i n}$ region mutations are described as $d p p^{H i n x}$, for those alleles that have a dominant haplo-lethal phenotype or $d p p^{h i n-2 x}$ for those alleles that are recessive lethals (see below). $d p p^{d i s k}$ region mutations, formerly $d p p^{x}$, are now termed $d p p^{d x}$ (where $x$ begins with a number) or $d p p^{d-y}$, where $y$ begins with a letter. Some exceptions to this standard set of conversions were required; they are noted in the text.

Unless otherwise noted, $d p p$ alleles are described in Spencer et al. (1982) or Segal and Gelbart (1985). Given the phenotypic and complementation analyses of $d p p^{4}$ and $d p p^{27}$ (Spencer et al. 1982) with $d p p^{H i n}$ alleles, we have renamed these mutations as

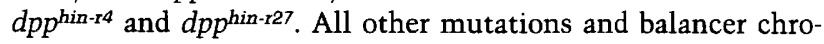
mosomes are described in Lindsley and Grell (1968). Dominant $d p p^{H i n}$ alleles are defined as haplo-lethal when resulting in less than $5 \%$ survival upon outcrossing to standard laboratory strains. The haplo-lethality of newly generated $d p p$ mutations was ascertained by testing the viability of flies bearing the lesion in question and only one additional normal copy of $d p p^{H i n+}$. Recessive embryonic lethal $d p p$ alleles have been identified by their homozygous embryonic lethality and by allelism to the tester alleles $d p p^{\text {hin-r4 }}$ and $d p p^{\text {hin-r27; }}$ such mutations are designated as $d p p^{\text {hin-r }}$ alleles.

We have used several insertional duplications which encompass the entire $d p p$ gene. $D p(2 ; 2) D T D 48$ and $D p(2 ; 3) D T D 33$ were initially described as carrying $d p p^{h o 2}$ (Spencer et al. 1982), but subsequent molecular analysis has shown that this allele is a reisolate of $d p p^{d-h o}$ (R. Blackman, unpubl.). These duplications are now designated as carrying $d p p^{d-h o}$. The $d p p^{\text {hin-r4 }}$ $D p(2 ; 2) M V D 1-D 2 d p p^{d-h o}$ chromosome is a previously undescribed rearrangement isolated in a transvection study; it carries In(2LR) 23F; 40 superimposed upon $\mathrm{Dp}(2 ; 2) 21 \mathrm{E} ; 25 \mathrm{~A} 1$ into 33B, and carries $d p p^{\text {hin-r4 }}$ in the normally situated $d p p$ copy and $d p p^{d-h o}$ in the duplicated copy.

Flies were cultured on standard Drosophila cornmeal yeast extract sucrose medium in $25-\mathrm{mm} \times 95-\mathrm{mm}$ shell vials or quarter-pint urine specimen bottles. All crosses were reared at $25^{\circ} \mathrm{C}$.

\section{Mutagenesis procedures}

Mutations in the $d p p^{H i n}$ region were recovered in three mutageneses. The first two types are similar in design. In the first screen, $d p p^{+} S p$ BI Dp(2;2)DTD48, $d p p^{d-h o} / \operatorname{In}(2 L R) C y O, C y$ $d p^{l v 1} \mathrm{pr} \mathrm{cn}^{2}$ males were irradiated with 4500 rads of X-rays and mated to multiply marked tester females carrying $d p p^{d \cdot h o}$ last $d p p^{d-h o}$ ed $d p$ cl). Of the genotype $d p p^{\star} S p$ BI Dp(2;2)DTD48, $d p p^{d-h o} / d p p^{d-h o}, 60,000 \mathrm{~F}^{1}$ progeny were screened for a heldout phenotype.

In mutagenesis 2 , males of the genotype $d p p^{+} S p \quad B 1$ $D p(2 ; 2) d p p^{d 21} /+$ were irradiated with 3300 rads of gamma rays and mated to tester $d p p^{d-h o}$ females. This mutagenesis differed from the first in two regards. First, $D p(2 ; 2) d p p^{d 21}$ is $d p p^{H i n+}$, but lacks the entire $d p p^{\text {disk }}$ region. Second, the tester chromosome included a rearrangement $[\operatorname{In}(2 \mathrm{LR}) 23 \mathrm{~A}$; $41 \mathrm{~A}$ ] that disrupts transvection within $d p p$ (Gelbart 1982). The presence of this rearrangement permitted the recovery of $d p p$ mutations that exhibit synapsis-dependent complementation with $d p p^{d-h o}$. The tester females were derived from a stock segregating two similar inversions: $\operatorname{In}(2 L R) D T D 18$, ast $d p p^{d-h o}$ ed $d p c 1$ and In(2LR)DTD21, ast dpp d.ho ed dp cl. In(2LR)DTD18 breaks in 22F3-23A1 and 41A, whereas In(2LR)DTD21 breaks in 23Al-2 and $41 \mathrm{~A}$. Both of these inversions were generated as transvection-disrupting rearrangements (Gelbart 1982). Approximately $65,000 F_{1}$ progeny bearing the duplicated chromosome were screened for heldout wings.

Mutagenesis 3 was an $\mathrm{F}_{2}$ lethal screen. Homozygous $d p p^{+} d p$ $c n b w$ males were treated with ethylmethane sulfonate (EMS) and mass mated to $D p(2 ; 1) G 146, d p p^{+} / \operatorname{In}(1) F M 7 a, y^{31 d} s c^{8} w^{a}$ vOf $B ; d p p^{H i n 48} S p c n / I n(2 L R) C y O, C y d p^{l v 1} p r c n^{2}$ females. Individual males bearing a balanced mutagenized second chromosome and the $\mathrm{X}$-linked duplication of $d p p^{+}$were mated to two $C(1) M 3, y^{2} / Y$; dpp hin-r4 Sco/In(2LR)CyO, Cy $d p^{l v 1}$ pr cn $\mathrm{cn}^{2}$ females. We assayed 2053 cultures for the absence of $\mathrm{Cy}^{+} \mathrm{fe}-$ males; potential haplo-lethal or recessive lethal $d p p$ alleles were recovered from the sibling $\mathrm{F}_{2}$ males and balanced.

$\mathrm{X}$-ray irradiation was performed with a Keleket $250-\mathrm{kV}$ X-ray machine at maximum voltage with no filter, at dose rates of 350-400 rads/min. Gamma-ray irradiation was performed with a ${ }^{137} \mathrm{Cs}$ GammaCell 10 source at $333 \mathrm{rads} / \mathrm{min}$. Males were aged for several days, irradiated, and mated to the appropriate females for two days. Males were then discarded, and inseminated females were allowed to lay eggs on fresh medium for three 2-day transfers.

EMS was administered to male flies according to the method of Lewis and Bacher (1968). Aged males were treated and allowed to mate with tester females for 1 day. Females were collected and allowed to lay eggs for three 2-day transfers.

\section{Determination of lethal phase}

Various lethal combinations were assayed for their lethal phase by determining frequencies of hatched embryos, pupae, and adults. Flies carrying the mutant allele were outcrossed to wild-type strains and the resulting heterozygous offspring were crossed to similarly outcrossed strains bearing the tester allele, to avoid homozygosity for extraneous lethals as well as to avoid lethality associated with aneuploidy caused by exchanges with the balancer inversions.

Dominant haplo-lethal mutations were initially recognized by their segregation behavior in test crosses. Lethal phases were determined for both $d p p^{\text {Hin- }} / d p p^{+}$and $d p p^{H i n-} / D f(2 L) d p p^{H i n-}$ combinations. Male flies bearing $d p p^{H i n-} / d p p^{+} D p(2 ; 2) D T D 48$, 
$d p p^{d-h o}$ were crossed to wild-type females, and at least 200 eggs were scored from each cross. Approximately $50 \%$ of the resulting zygotes from these crosses died during embryogenesis, and no other lethal phase was detectable. The lethal phase of null combinations was determined by crossing $d p p^{\text {Hin- }} / d p p^{+}$ $D p(2 ; 2) D T D 48$, $d p p^{d-h o}$ males to females carrying $d p p^{\text {hin-r4 }}$ $D p(2 ; 2) M V D 1-D 2, d p p^{d-h o} / D f(2 L) D T D 2, d p p^{\text {Hin }-}$. At least 200 eggs were scored from each of these crosses, with $25-28 \%$ of the zygotes dying during embryogenesis. No larval or pupal mortality was detectable.

Recessive lethal mutations were initially recognized by their ability to survive in a heterozygous state without duplications of $d p p^{H i n+}$. Lethal phases for recessive lethal alleles were determined by reciprocal test crosses. Flies bearing either the newly isolated or tester lesions were outcrossed to wild-type strains, and the resulting progeny were intercrossed. Tester alleles were either $d p p^{\text {hin-14 }}$ or $d p p^{\text {hin-r27 }}$ (Spencer et al. 1982). In all cases, approximately $25 \%$ of the resulting zygotes died during embryogenesis, with no other detectable lethal phase.

\section{Germ line mitotic recombination}

Fs(2)1 is a dominant germ line autonomous female sterile mutation mapping on chromosome arm $2 \mathrm{~L}$ between aristaless (2-0.4) and dumpy (2-13.0) (J. Szabad, pers. comm.). Heterozygous females lay a few flaccid eggs per day, which are easily distinguishable from morphologically normal eggs. Heterozygous females bearing germ line clones are recovered at a frequency of about $6-7 \%$ upon adult irradiation with 1500 rads (J). Szabad, pers. comm.).

Germ line clones were induced by irradiating larvae resulting from a cross of $d p p^{H i n 88} d p$ cn $b w / I n(2 L R) S M 6 a, C y$; $D p(2 ; 3) D T D 33, d p p^{d-h o}$ red e/Tp(3;3)MKRS, kar ry ${ }^{2} S b$ females to $F s(2) 1 / C y$ Roi males. Tp (3;3)MKRS is a third chromosome that balances proximal and medial chromosome arm $3 \mathrm{R}$ and that carries the dominant marker Stubble (Hilliker et al. 1980). Clones were recovered in irradiated female escapers of the genotype $d p p^{H i n 88} d p$ cn bw/Fs(2) 1 ; $/ T p(3 ; 3) M K R S$, kar ry ${ }^{2} S b$ with egg production monitored for 15 days. Clonally derived eggs were scored $24-48 \mathrm{hr}$ after oviposition for hatchability and for the lethal phenotype. Hatched larvae were grown to adulthood and test crossed for the presence of the expected marker mutations.

\section{Embryonic phenotypes}

Embryos were aged to $24-30 \mathrm{hr}$ and dechorionated $5 \mathrm{~min}$ in $50 \%$ sodium hypochlorite. After extensive washing with $\mathrm{H}_{2} \mathrm{O}$, the embryos were suspended in a mix of $10 \mathrm{ml}$ heptane : $9 \mathrm{ml}$ methanol : $1 \mathrm{ml} 0.5 \mathrm{M}$ EGTA, $\mathrm{pH} 8.0\left(4^{\circ} \mathrm{C}\right)$ to remove the vitelline membranes (M. Akam, pers. comm.). The embryos were collected out of the lower phase and rehydrated in a decreasing ethanol series. They were then treated with 4 parts acetic acid : 1 part glycerol and mounted in Hoyer's mounting medium (Van der Meer 1977).

Gastrulation was observed with bright-field or phase optics in embryos that had been covered in a thin layer of Voltalef $3 \mathrm{~S}$ oil. Putative mutant and wild-type eggs were followed until the cuticular phenotypes could be determined.

\section{Acknowledgments}

We wish to thank R. Daniel St. Johnston for his critical comments on the manuscript and both Macy Koehler and Julia Smith for their excellent job of preparing the figures. This work was supported by a National Institutes of Health (NIH) grant to
W.M.G. (GM 28669). During the course of this work, V.F.I. was an NIH Predoctoral Trainee in Genetics.

\section{References}

Anderson, K.V. and C. Nüsslein-Volhard. 1984a. Information for the dorsal-ventral pattern of the Drosophila embryo is stored as maternal mRNA. Nature 311: 223-227.

- 1984b. Genetic analysis of dorsal-ventral embryonic pattern in Drosophila, in Pattern formation: A primer in developmental biology. (ed. G. Malacinski and S. Bryant), MacMillan Inc., New York.

Anderson, K.V., L. Bokla, and C. Nüsslein-Volhard. 1985a. Establishment of dorsal-ventral polarity in the Drosophila embryo: The induction of polarity by the Toll gene product. Cell 42: 791-798.

Anderson, K.V., G. Jurgens, and C. Nüsslein-Volhard. 1985b. Establishment of dorsal-ventral polarity in the Drosophila embryo: Genetic studies on the role of the Toll gene product. Cell 42: 779-789.

Blackman, R.K., R. Grimaila, M.M.D. Koehler, and W.M. Gelbart. 1987. Mobilization of hobo elements residing within the decapentaplegic gene complex: Suggestion of a new hybrid dysgenesis system in Drosophila melanogaster. Cell 49: 497-505.

Campos-Ortega, J. 1983. Topological specificity of phenotype expression of neurogenic mutations in Drosophila. Wilhelm Roux's Arch. Dev. Biol. 192: 317-326.

Campos-Ortega, J. and V. Hartenstein. 1985. The embryonic development of Drosophila melanogaster. Springer-Verlag, Berlin.

Frohnhofer, H.G. 1982. Abgrenzung maternaler und zygotischer Anteile bei der genetischen Kontrolle der Musterbildung in Drosophila melanogaster: Vier Mutanten, die zygotisch das dorsoventrale Muster des Embryos storen, zeigen keinen maternalen Effekt in homozygoten Keimbahnklonen. Diplomarbeit, Universität Tübingen, FRG.

Gelbart, W.M. 1982. Synapsis-dependent allelic complementation at the decapentaplegic gene complex in Drosophila melanogaster. Proc. Natl. Acad. Sci. 79: 2636-2641.

Gelbart, W.M., V.F. Irish, R.D. St. Johnston, F.M. Hoffmann, R. Blackman, D. Segal, L.M. Posakony, and R. Grimaila. 1985. The decapentaplegic gene complex in Drosophila melanogaster. Cold Spring Harbor Symp. Quant. Biol. 50: 119125.

Hilliker, A.J., S.H. Clark, A. Chovnick, and W.M. Gelbart. 1980. Cytogenetic analysis of the chromosomal region immediately adjacent to the rosy locus in Drosophila melanogaster. Genetics 95: 95-110.

Irish, V.F. 1986. Embryonic functions of the decapentaplegic gene complex in Drosophila melanogaster. Ph.D. thesis, Harvard University.

Jurgens, G., E. Wieschaus, C. Nüsslein-Volhard, and H. Kluding. 1984. Mutations affecting the larval cuticle in Drosophila melanogaster. II. Zygotic loci on the third chromosome. Wilhelm Roux's Arch. Dev. Biol. 193: 283-295.

Lewis, E.B. and F. Bacher. 1968. Method for feeding ethylmethane sulfonate (EMS) to Drosophila males. Dros. Inform. Serv. 43: 193.

Lindsley, D. and E. Grell. 1968. Genetic variation of Drosophila melanogaster. Carnegie Institution of Washington, Publ. No. 627.

Lohs-Schardin, M., C. Cremer, and C. Nüsslein-Volhard. 1979. A fate map for the larval epidermis of Drosophila melanogaster: Localized cuticle defects following irradiation of the blastoderm with an ultraviolet laser microbeam. Dev. Biol. 73: 239-255. 
Nüsslein-Volhard, C., M. Lohs-Schardin, K. Sander, and C. Cremer. 1980. A dorso-ventral shift of embryonic primordia in a new maternal effect mutant of Drosophila. Nature 283: 474-476.

Nüsslein-Volhard, C., E. Wieschaus, and H. Kluding. 1984. Mutations affecting the pattern of the larval cuticle in Drosophila melanogaster. I. Zygotic loci on the second chromosome. Wilhelm Roux's Arch. Dev. Biol. 193: 267-282.

Padgett, R.W., R.D. St. Johnston, and W.M. Gelbart. 1987. A transcript from a Drosophila pattern gene predicts a protein homologous to the transforming growth factor- $\beta$ gene family. Nature 325: 81-84.

Segal, D. and W.M. Gelbart. 1985. Shortvein, a new component of the decapentaplegic gene complex in Drosophila melanogaster. Genetics 109: 119-143.

Simpson, P. 1983. Maternal-zygotic gene interactions during formation of the dorso-ventral pattern in Drosophila embryos. Genetics 105: 615-632.

Spencer, F.A., F.M. Hoffmann, and W.M. Gelbart. 1982. Decapentaplegic: A gene complex affecting morphogenesis in Drosophila melanogaster. Cell 28: 451-461.

St. Johnston, R.D. and W.M. Gelbart. 1987. Decapentaplegic transcripts are localized along the dorsal-ventral axis of the Drosophila embryo. EMBO J. 6: 2785-2791.

Struhl, G. 1984. Splitting the bithorax complex of Drosophila. Nature 208: 454-457.

Technau, G.M. and J.A. Campos-Ortega. 1986. Lineage analysis of transplanted individual cells in embryos of Drosophila melanogaster. II. Commitment and proliferative capabilities of neural and epidermal cell progenitors. Wilhelm Roux's Arch. Dev. Biol. 195: 445-454.

Van der Meer, J.M. 1977. Optically clean and permanent whole mount preparations for phase-contrast microscopy of cuticular structures of insect larvae. Dros. Inform. Serv. 52: 60.

Wakimoto, B.T., F.R. Turner, and T.C. Kaufman. 1984. Defects in embryogenesis in mutants associated with the Antennapedia gene complex in Drosophila melanogaster. Dev. Biol. 102: $147-172$.

Wieschaus, E., C. Audit, and M. Masson. 1981. A clonal analysis of the roles of somatic cells and germ-line during oögenesis in Drosophila. Dev. Biol. 88: 92-103.

Wu, C.-T. 1984. A genetic analysis of transvection in Drosophila melanogaster. Ph.D. thesis, Harvard University. 


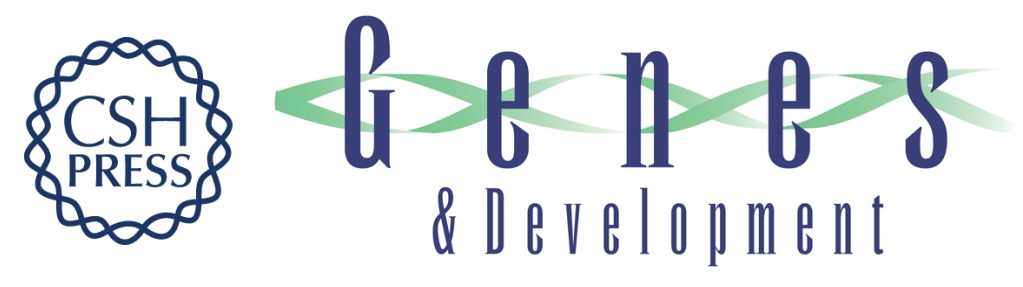

\section{The decapentaplegic gene is required for dorsal-ventral patterning of the Drosophila embryo.}

V F Irish and W M Gelbart

Genes Dev. 1987, 1:

Access the most recent version at doi:10.1101/gad.1.8.868

References This article cites 23 articles, 5 of which can be accessed free at: http://genesdev.cshlp.org/content/1/8/868.full.html\#ref-list-1

License

Email Alerting

Receive free email alerts when new articles cite this article - sign up in the box at the top Service right corner of the article or click here.

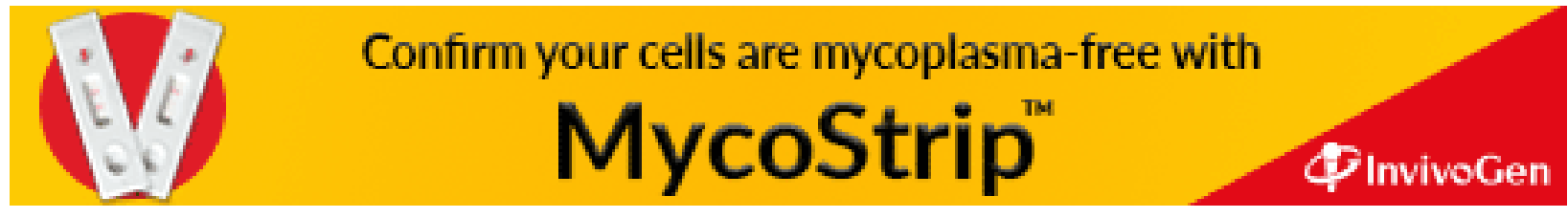

